\title{
Research article \\ The effectiveness of craniosacral therapy compared to standard treatment for pelvic girdle pain during pregnancy: A quasi experimental study
}

\author{
K. Kotteeswaran ${ }^{1}$, V. Meena ${ }^{2}$, B. Sathish Kumar ${ }^{2}$, Tamil Selvi R. ${ }^{3}$, Pavithira S. K. ${ }^{3}$ \\ ${ }^{1}$ Professor, ${ }^{2}$ Postgraduate Student, ${ }^{3}$ Undergraduate Student, Saveetha College of Physiotherapy, Saveetha Institute of Medical \\ and Technical Sciences, Chennai-602105, Tamil Nadu, India
}

(Received: May 2020

Revised: November 2021

Accepted: November 2021)

Corresponding author: K. Kotteeswaran. Email: k.kotteeswaran@gmail.com

\begin{abstract}
Introduction and Aim: Pelvic girdle pain is one of major pregnancy discomforts affecting about 50\% of pregnant women. There is no evidence that standard treatment is completely effective for pelvic girdle pain in pregnant women. Hence there is a requirement for newer safer therapies such as craniosacral therapy. The aim of this study is to evaluate effectiveness of craniosacral therapy in reducing pain and improving daily life activities which is restricted in pregnant women with pelvic girdle pain.

Materials and Methods: In this quasi-experimental study, we recruited 30 pregnant women who were clinically diagnosed with pelvic girdle pain using convenient sampling technique. They were divided into control and craniosacral therapy treated group. Using NPRS and PGQ, their pain intensity and disability values were recorded and compared pre and post treatment using mean and standard deviation. The paired ' $t$ ' test was used for statistical analysis.
\end{abstract}

Results: Group A post treatment NPRS value range was mean $=3.00, \mathrm{SD}=0.85$ and PGQ post treatment was mean $=36.87, \mathrm{SD}=4.91$. Group B post treatment NPRS value mean $=1.87, \mathrm{SD}=0.83$ and PGQ post treatment value was mean $=26.53, \mathrm{SD}=4.42$. Since between group significant difference was $\mathrm{p}<0.0001$ it was statistically significant.

Conclusion: Craniosacral therapy was found to be effective than standard treatment for pelvic girdle pain during pregnancy

Keywords: Pelvic girdle pain; craniosacral therapy, pregnant women, posterior pelvic provocation test.

\section{INTRODUCTION}

$\mathrm{P}$ elvic girdle pain is defined as the pain experienced between the posterior iliac crest and the gluteal region, in particularly in the vicinity of the sacroiliac joints. The pain may radiate up to the posterior thigh and can also occur in conjunction with or separately in the symphysis Joint. Pelvic girdle pain is one of major pregnancy discomforts affecting about $50 \%$ of pregnant women (1). It is characterized by pain, instability, limitations of mobility and functioning in pelvic girdle region.

The degree of pain and discomfort varies from each pregnant woman. Based on the degree and the instability of the pelvis it can be classified under three levels. In Type 1, the women will have no complaints regarding difficulty in performing daily life activities and this is biomechanically because the pelvic ligaments support the pelvis sufficiently. In type 2 the patient experiences pain and weakness while performing daily life activities and this is biomechanically because the pelvic ligaments does not support the pelvis sufficiently. Type 3 is similar to type 2 , but it is very severe that even exercise program or any medical treatment cannot resolve it. In addition to pain the women feel stressed and will be unable to do daily activities, takes constant leave at work. The average sick leave is about 7 to 12 weeks (2).

The risk factors for pelvic girdle pain during pregnancy are previous history of low back pain (or) trauma in the back/pelvis, higher level of stress and strenuous work (3). Pelvic girdle pain occurs due to increased strain on back and pelvic muscles during pregnancy. During pregnancy there is instability of pelvic joints. As a compensatory mechanism, the muscles (abdominals, pelvic floor, gluteal and paraspinal muscles) work harder results in pain and stiffness leading to pelvic girdle pain. A person with pelvic girdle pain often experiences pain and difficulty while sitting and standing. In few patients it is associated with swelling or inflammation over the joint or clicking sound while walking. For few it is associated with bowel and bladder dysfunction or waddling gait. Due to the mal alignment of the pelvic joints the person's back is usually stooped while standing. Pelvic girdle pain is often misdiagnosed as low back pain. Posterior pelvic provocation test helps to differentiate pelvic girdle pain from low back pain. Other test which is used in the diagnoses of pelvic girdle pain are Modified Trendelenburg test, palpation of long dorsal ligament and symphysis pubis and active straight leg raising test (4). 
The commonly used scales to measure the intensity of pain and disability in persons with of pelvic girdle pain are NPRS and PGQ. NPRS measures the intensity of pain in centimeters, and it is the most reliable and valid scale with correlations ranging from 0.86 to 0.95 (5). PGQ is a condition specific scale that measures the disability in percentage, and it is highly reliable and valid with correlations ranging from 0.86 to 0.96 . Treatment is essential in women with pelvic girdle pain since the condition persists even postpartum. The average time to recover is about 6.25 years and can extend even more based on the severity. Medical management for treatment of pelvic girdle pain includes prescription of drugs such as NSAIDs but those are not safe after 30 weeks of pregnancy (6). The common therapies used for pelvic girdle pain during pregnancy are usually exercises, manual therapy (joint mobilization and manipulation), pelvic belts, massage, therapeutic ultrasound, and TENS. Treatment is essential since the residual symptoms persist even after pregnancy (7).

To my knowledge, currently there is no evidence that standard treatment is completely effective for pelvic girdle pain in pregnant women. The treatment is still questionable. Hence there is requirement for newer safer therapies such as craniosacral therapy. Craniosacral therapy is one type of complementary alternative technique which was developed by John Upledger in the 1970s. Upledger stated that craniosacral therapy can be used to treat various conditions such as back pain, neck pain, fibromyalgia, migraine, scoliosis, and Alzheimer's disease. It is a common treatment used by osteopaths either alone or combined with other treatments. It is a form of gentle "hands on" clinical skill with claimed clinical effect of releasing tension in fascia, ligaments, and muscles in the sacral region. The reason for using this technique is that it is more natural and safer compared with pharmaceutical drugs.

The mechanism involved is that sensory stimulation leads to inhibition of pain transmission at spinal or central level (8). It mainly involves the stimulation of large diameter $\mathrm{C}$ fibers by Touch to inhibit pain carried by the small diameter $A \beta$ fibers. Other proven effects of craniosacral therapy were decreasing tone of intrafascial muscle cells, decreasing muscle tension, and increasing parasympathetic nervous system response. All these in turn promote flexibility and mobility of the connective tissue which improves mobility of joints.

\section{MATERIAL AND METHODS}

\section{Patients and data collection}

Total of 30 pregnant women were selected using convenient sampling technique from Physiotherapy outpatient department, Saveetha Medical College
Hospital, Chennai based on the inclusion criteria which includes Healthy pregnant women between 2230 gestational weeks diagnosed with bilateral pelvic girdle pain, Pregnant women with moderate pelvic girdle pain as measured by Numerical Pain Rating Scale (NPRS) within the range of 4-6 and Pregnant women with disability level between $20-70 \%$ as measured by pelvic girdle questionnaire (PGQ) and exclusion criteria which is women with other pain conditions, orthopedic or systemic illness or with any previous history of miscarriage or abortion.

Pregnant women were diagnosed with pelvic girdle pain based on the following criteria: (a) history of pain between the posterior iliac crests and the gluteal region. (b) pain arising while palpating symphysis pubis (Patient was asked to lie in supine position and pubis symphysis was palpated and hand was withdrawn. If the pain persisted for more than 5 seconds even after withdrawal then the test is positive). (c) Modified Trendelenburg test is positive (Patient was asked to stand on one leg for 30 seconds, if balance was an issue, they were asked to hold on to something for support. Test is positive if pelvis drops on the unsupported side). (d) Positive posterior pelvic provocation test (P4) (The patient was made to lie in supine position and hip was flexed to 90 degrees. One hand was placed under the sacrum and the other hand was placed on the femur and 3-6 velocity thrust pressure was applied. Test is positive if it provokes pain).

All criteria had to be fulfilled for the diagnosis and out of which posterior pelvic provocation test confirmed and differentiated pelvic girdle pain from low back pain. The entire test which was performed in supine position was no longer done more than 20 seconds. All the tests were performed in a safe and comfortable manner.

The study was explained to the patients and written consent was obtained from the subjects. The participants were asked to fill the pelvic girdle questionnaire. The person who fulfilled all the criteria was then allocated to either control group or craniosacral therapy group. Control group consisted of 15 subjects who received standard treatment and hot pack therapy. Craniosacral therapy group consisted of 15 subjects who received craniosacral therapy and hot pack therapy. If the exercises aggravated the pelvic pain the participants were advised to contact investigator for further instruction.

\section{Control group (Group A)}

The control group was provided with Postural advice which included standing tall with relaxed shoulders, holding head up straight with chin in and standing such that the weight is equally distributed on both feet and avoid standing on one foot and leaning on one side. And while sitting they were advised to sit erect and use cushion to support back and sit such 
that the weight distributes equally on both the hips. They were advised to keep their foot flat or on small stool and avoid slouching and crossing legs while sitting.

Do and Don'ts during pregnancy were advised which included avoid lifting weights, avoid bending forwards while standing, always lie on the side with pillows between knees and behind the back, avoid wearing shoes with heel and change position frequently (every 10-15 minutes). Home training program which included abdominal and gluteal muscle strengthening exercises such as Pelvic tilts: The patient was asked to sit tall on the swiss ball with good posture (shoulder relaxed, spine straight, chest lifted, and abdominals gently pulled inwards). The pelvis was tilted forward and backward such that there is rounding and lengthening of the lumbar spine (9).

Repetition 10 times continuously, side lying knee lifts: The patient was asked to lie on the side with shoulders, hips and knees lined up straight. The patient was asked to support themselves by placing their arms in front of them. The leg was slowly lifted as high and as comfortable, and the patient was asked to breathe normally. Repetition: 10 times, Hold time: 20 seconds, Rest period: 15 to 20 seconds between each repetition. Tailor sitting: The patient was asked to sit on the mat with legs apart and heels close together. They felt stretch in the inner side of the thigh. They were asked to maintain this position for few seconds and then relax and repeat. Repetition: 5 times, Hold time: 5 to 10 seconds, Rest period: 10 seconds between each repetition.

\section{Craniosacral therapy group (Group B)}

The patient was asked to lie inside lying position and pillows were placed for comfort. Hands were placed bilaterally under the lower lumbar region and L5 S1 release, sacroiliac release and superior and inferior pubic symphysis release was done using gentle fascial traction, release, and unwinding techniques with the respective palpated joint restrictions. L5 - S1 release: The patient was made to lie inside lying position with knees bent. One hand was placed under the spinous process of L5 and another on the patient's knee. Perpendicular pressure was applied over the L5 by the therapist by leaning back. While leaning back, the patient's knee was moved away by the therapist to cause a counter torque. This technique was continued until L5 - S1 is released. Sacroiliac release: The patient was made to lie inside lying position with knees straight and pillows in their knees. One hand was placed over the sacrum and pressure was applied and counter pressure was applied over the ilium. This technique was continued until the sacroiliac is released. Superior and inferior pubic symphysis release: The therapist hand was placed over the pubic symphysis and release was done by gliding it superiorly and inferiorly. Palpating restrictions and releasing: The fascia around the sacrum, ilium, and sacroiliac joint and pubic symphysis were palpated with patients inside lying position. Deep palpation was done, and restrictions were released by applying pressure over the restriction for 10 seconds to two minutes and followed to the end where it remains free. This technique was done until patient felt less pain. Duration: 20-30 minutes split into two sessions with rest period. 1stsession was for about 10-15 minutes by one researcher and 2ndsession was for about 1015 minutes by another researcher. Rest period between two sessions was about 2 minutes. Total treatment duration: Thrice a week for two weeks (6 treatment sessions).

\section{Hot pack therapy}

Both the groups were treated with hot pack therapy as a common treatment. The patient was positioned comfortably inside lying position. The part to be treated was exposed. Hot pack was wrapped around a towel and applied over the lower back and pelvic region for the duration of 15-20 minutes.

\section{Outcome measures}

Numerical pain rating scale (NPRS) - measures the pain intensity in centimeters $(\mathrm{cm})$. The participants were included in the study by asking to rate the pain intensity from $0-10 \mathrm{~cm}$ and was selected if they had pain intensity between $4-6 \mathrm{~cm}$. The value was recorded and after the provision of treatment for two weeks and at the follow up visit the pregnant women were again asked to rate the pain intensity. These values were also recorded for further statistical analysis. Pelvic girdle questionnaire (PGQ) measures the disability in percentage (\%). Assess activity limitation and symptoms in patients with pelvic girdle pain. The participants were included in the study by asking to fill the PGQ and if the calculated disability level was between $20-70 \%$. The value was recorded and after the provision of treatment for two weeks and at the follow up visit the pregnant women were again asked to fill the questionnaire and the disability level was calculated. These values were also recorded for further statistical analysis.

\section{Statistical analysis}

The collected data was tabulated and analyzed using descriptive $\&$ inferential statistics. To all parameters mean and standard deviation (SD) was used. Paired ttest was used to analyze significant changes between pre- and post-treatment measurements. Unpaired ttest was used to analyze significant changes between groups for both the scales. $\mathrm{P}$ value $<0.0001$ was considered as statistically significant. 


\section{RESULTS}

Table 1: Comparison of NPRS value pre and post treatment for control group

Interpretation of Results

\begin{tabular}{|c|c|c|c|c|}
\hline NPRS (cm) & Mean (cm) & SD $(\mathbf{c m})$ & t - value & p - value \\
\cline { 1 - 2 } Pre-test (At 0 week) & 5.00 & 0.76 & & \\
\cline { 1 - 2 } Post-test (At 2 ${ }^{\text {nd }}$ week) & 3.00 & 0.85 & 8.3666 & $<0.0001$ \\
\hline
\end{tabular}

In group A, the mean NPRS value pretest was 5.00 and posttest is $3.00 \mathrm{~cm}$. The standard deviation value for pretest is $0.73 \mathrm{~cm}$ and post-test are $0.85 \mathrm{~cm}$. The $\mathrm{t}$

value is 8.3666 and $p$ value was $<0.0001$ which was statistically significant (Table 1, Fig. 1).

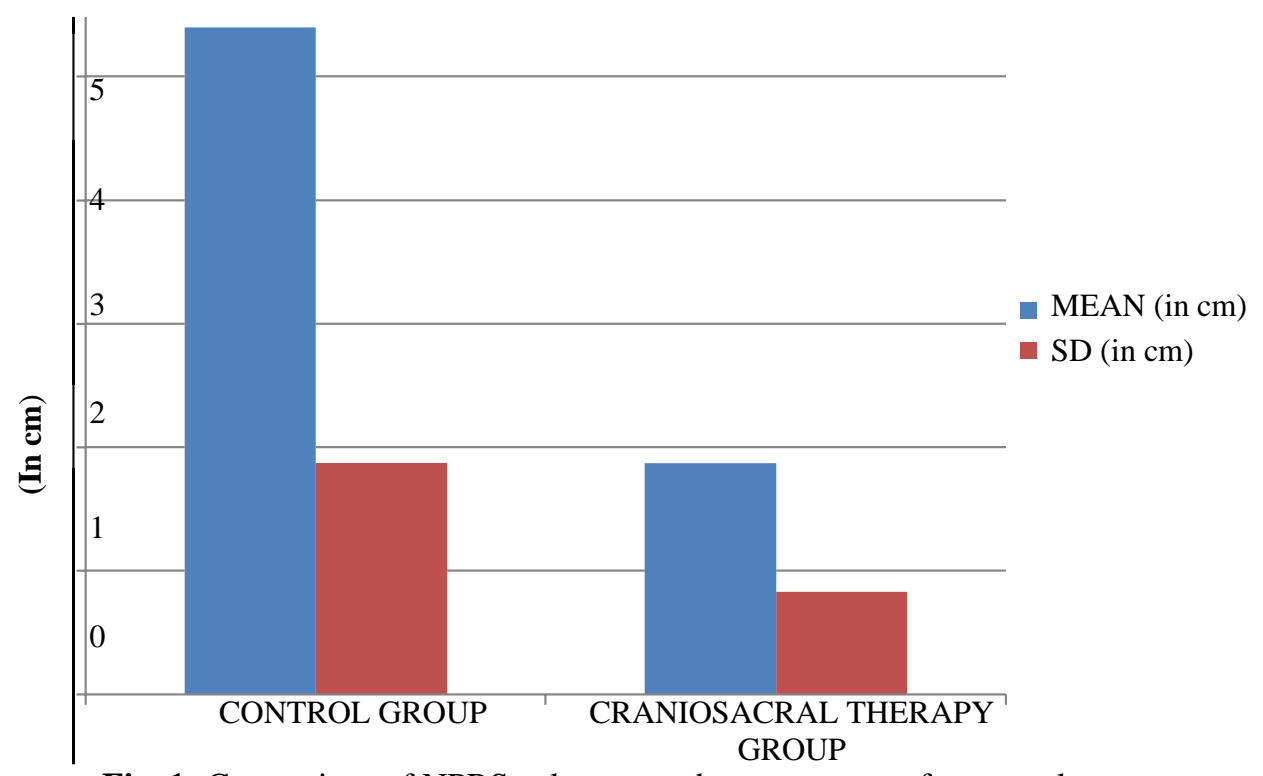

Fig. 1: Comparison of NPRS value pre and post treatment for control group

Table 2: Comparison of PGQ value pre and post treatment for control group Interpretation of Results:

\begin{tabular}{|c|c|c|c|c|}
\hline PGQ (in \%) & Mean (in \%) & SD (in \%) & t - value & p - value \\
\hline Pretest (At 0 week) & 48.73 & 5.98 & & \\
\cline { 1 - 3 } Post-test (At 2 (nd $^{\text {week) }}$ & 36.87 & 4.91 & 6.2972 & $<0.0001$ \\
\hline
\end{tabular}

In Group A, the mean PGQ value pre-test was $48.73 \%$ and posttest is $36.87 \%$. The standard deviation value for pre -test is $5.98 \%$ and posttest is
$4.91 \%$. The $\mathrm{t}$ value is 6.2972 and $\mathrm{p}$ value was $<0.0001$ which was statistically significant (Table 2, Fig. 2).

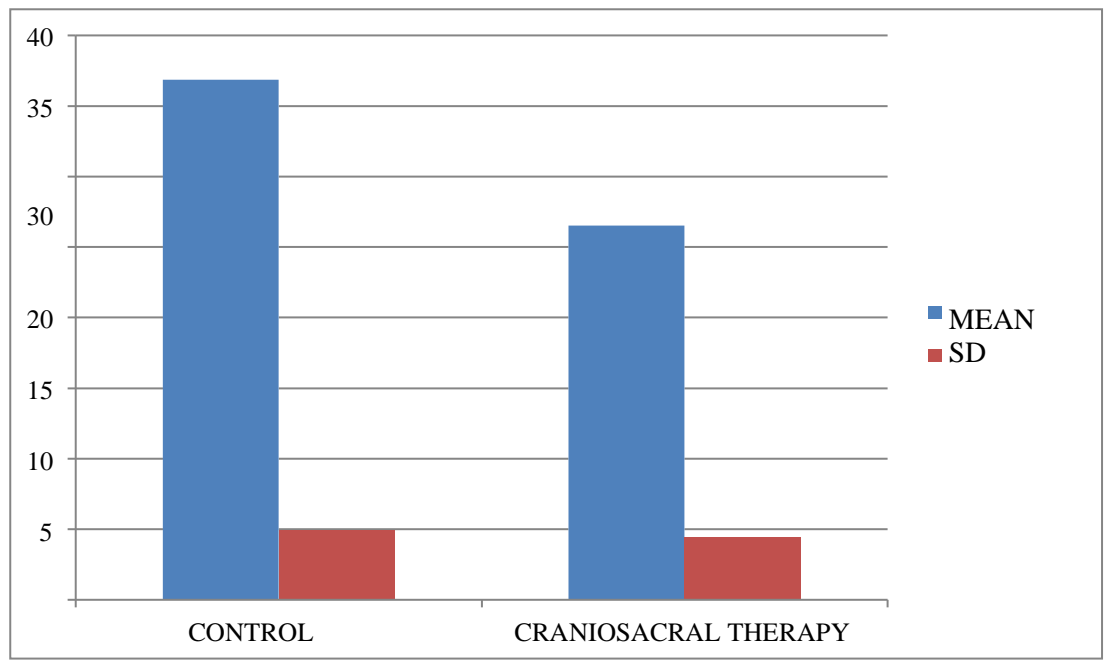

Fig. 2: Comparison of PGQ value pre and post treatment for control group

Table 3: Comparison of NPRS value pre and post treatment for craniosacral therapy group:

\begin{tabular}{|c|c|c|c|c|}
\hline NPRS (cm) & $\operatorname{Mean}(\mathrm{cm})$ & SD (cm) & t value & p value \\
\hline Pretest (At 0 week) & 5.40 & 0.74 & \multirow[b]{2}{*}{12.1590} & \multirow[b]{2}{*}{$<0.0001$} \\
\hline Post-test (At 2 $2^{\text {nd }}$ week) & 1.87 & 0.83 & & \\
\hline
\end{tabular}


In Group B, the mean NPRS value pre-test was 5.40 $\mathrm{cm}$ and post-test were $1.87 \mathrm{~cm}$ the standard deviation value for pretest is $0.74 \mathrm{~cm}$ and posttest are $0.83 \mathrm{~cm}$.
The $\mathrm{t}$ value is 12.1590 and $\mathrm{p}$ value was $<0.0001$ which was statistically significant (Table 3, Fig. 3).

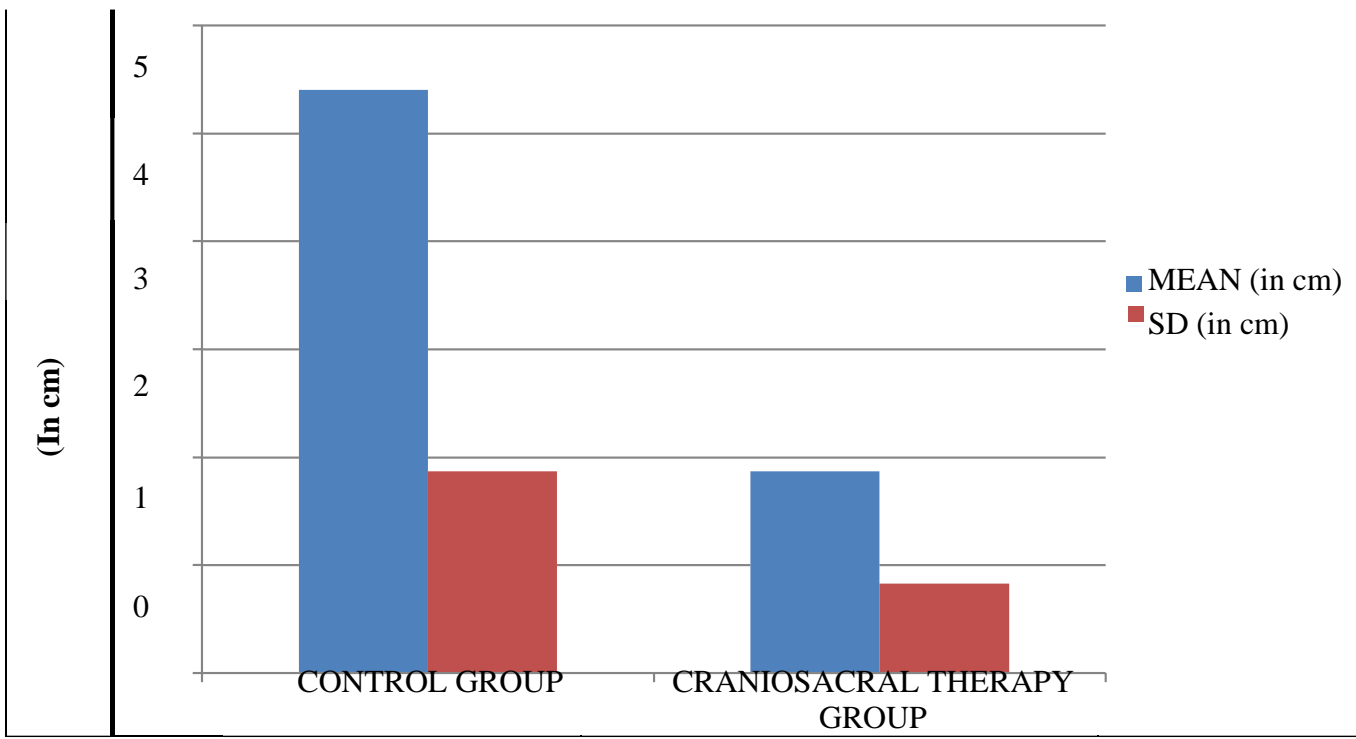

Fig. 3: Comparison of NPRS value pre- and post-treatment for craniosacral therapy group

Table 4: Comparison of PGQ value pre and post treatment for craniosacral therapy group:

\begin{tabular}{|c|c|c|c|c|}
\hline PGQ (in \%) & Mean (in \%) & SD (in \%) & t Value & p Value \\
\hline Pretest (At 0 week) & 52.07 & 6.03 & & \\
\cline { 1 - 3 } Post-test (At 2 $^{\text {nd }}$ week) & 26.53 & 4.42 & 12.1590 & $<0.0001$ \\
\hline
\end{tabular}

In Group B, the mean PGQ value pretest was $52.07 \%$ and posttest is $26.53 \%$. The standard deviation value for pretest is $6.03 \%$ and posttest is $4.42 \%$. The $\mathrm{t}$

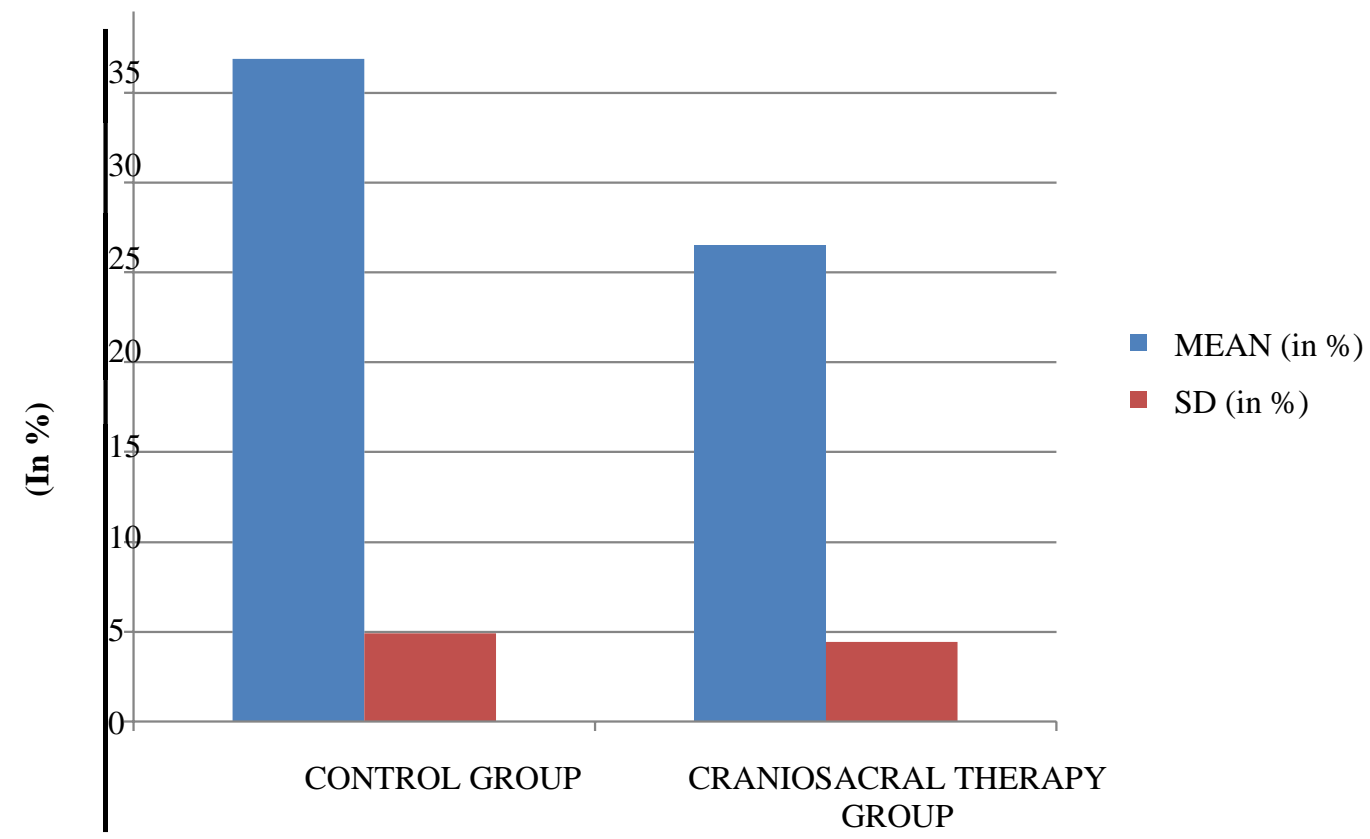

Fig. 4: Comparison of PGQ value pre- and post-treatment for craniosacral therapy group

Table 5: Comparison between control group and craniosacral therapy group for NPRS

\begin{tabular}{|c|c|c|c|c|}
\hline NPRS (cm) & Mean $(\mathbf{c m})$ & SD $(\mathbf{c m})$ & t Value & p Value \\
\hline $\begin{array}{c}\text { Control group } \\
\begin{array}{c}\text { Craniosacral } \\
\text { therapy group }\end{array}\end{array}$ & 3.00 & 0.85 & 3.6972 & $<0.0001$ \\
\hline
\end{tabular}

For between groups, the mean NPRS value for Group A was $3.00 \mathrm{~cm}$ and Group B was $1.87 \mathrm{~cm}$. The standard deviation value for Group A was $0.85 \mathrm{~cm}$ value is 11.2919 and $p$ value was $<0.0001$ which was statistically significant (Table 4, Fig. 4). 


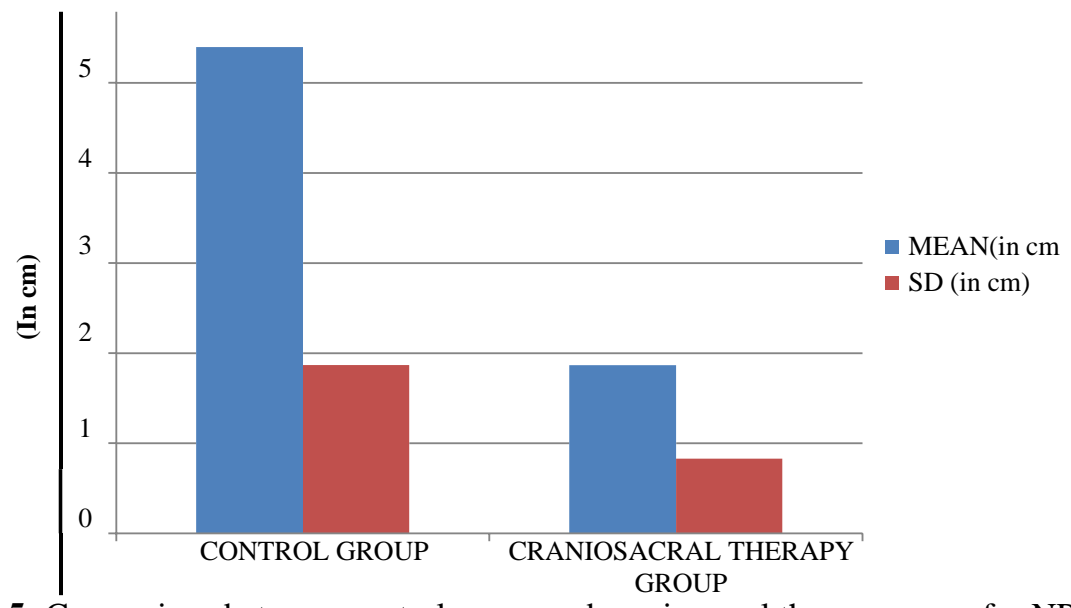

Fig. 5: Comparison between control group and craniosacral therapy group for NPRS

Table 6: Comparison between control group and craniosacral therapy group for PGQ

\begin{tabular}{|c|c|c|c|c|}
\hline PGQ (in \%) & Mean (in \%) & SD (in \%) & t value & p value \\
\hline Control group & 36.87 & 4.91 & & \\
\cline { 1 - 3 } $\begin{array}{c}\text { Craniosacral therapy } \\
\text { group }\end{array}$ & 26.53 & 4.42 & 6.0557 & $<0.0001$ \\
\hline
\end{tabular}

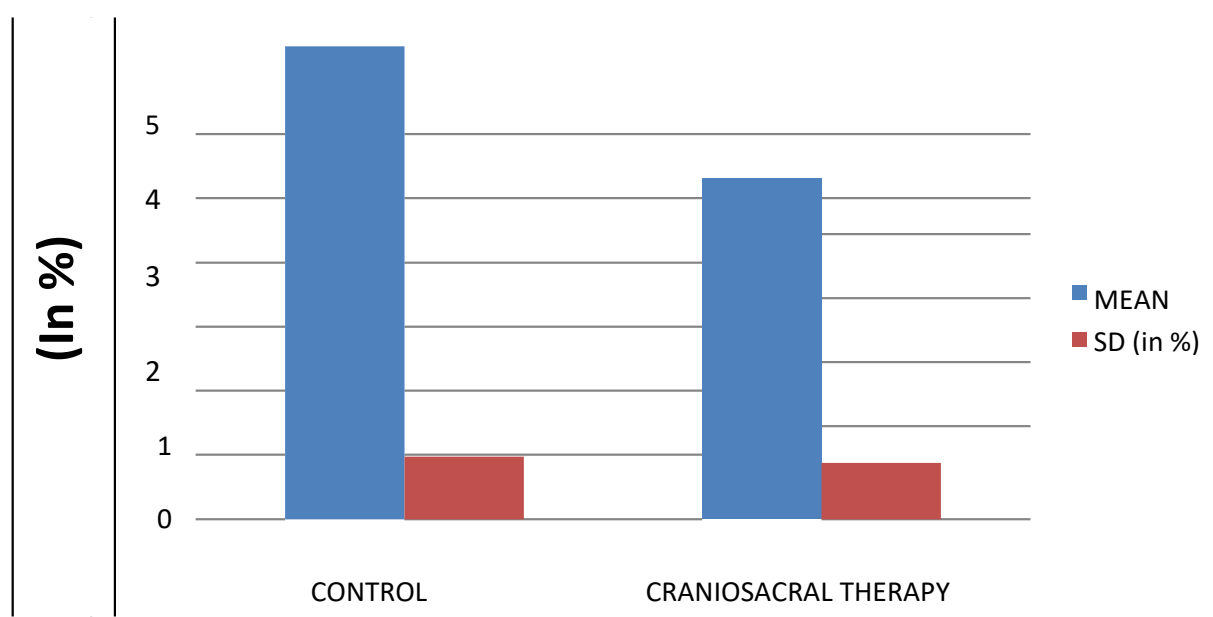

Fig. 6: Comparison between control group and craniosacral therapy group for PGQ

Between the group comparisons, the mean PGQ value for Group A was $36.87 \%$ and Group B was 26.53\%. The standard deviation value for Group A was $4.91 \%$ and Group B was $4.42 \%$. The $t$ value is 6.0557 and $\mathrm{p}$ value was $<0.0001$ which was statistically significant. (Table 6, Fig. 6). Comparison of Craniosacral therapy group and control group, statistical difference for pain reduction and reduced disability index was improved in Group B.

\section{DISCUSSION}

The main finding from this study was that there is reduced pain intensity and less functional deterioration among the women who received craniosacral therapy compared with women who received standard treatment. Our hypothesis on the mechanism of craniosacral therapy for reducing pain intensity is based on Melzack and Wall theory which states that stimulation of $\mathrm{A} \beta$ fibers inhibits pain which is carried by $\mathrm{C}$ fibers (10). Craniosacral therapy involves "Touch" which stimulates the $A \beta$ fibers. Stimulation of mechanoreceptors inhibits pain transmission at spinal level. Other proven effects of craniosacral therapy were decreasing tone of intrafascial muscle cells, decreasing muscle tension, and increasing parasympathetic nervous system response (11). All these in turn promote flexibility and mobility of the connective tissue which improves mobility of joints. This mechanism has happened in pregnant women who received craniosacral therapy and increased their pelvic mobility and reduced the laxity of sacroiliac joint.

Similar results were proved in two previous studies and had same conclusion which suggests that craniosacral therapy has pain-relieving effect and halts the deterioration of function $(12,13)$. A 2010 study examined the effects of craniosacral therapy on patients with fibromyalgia and concluded that it showed effects on decreasing pain, improving quality of life and reducing anxiety. Few studies suggest that effects of pain relief, maybe initiating placeboelicited inhibition of pain (14). Pain reduced due to such placebo effect, and we informed the participants that the study was designed to compare craniosacral therapy with standard treatment. This further minimized bias and indicates our intervention was successful though there was neutral presentation. 
Women who received craniosacral therapy claimed that they experienced deeper state of relaxation. They had fewer symptoms and found treatment to be helpful. Findings from this study also indicates "practitioner effect" exists since there is direct relationship is established between the therapist and the patient. Though the pain and disability of the pregnant women who received craniosacral therapy did not completely reduce or they fully recovered from pelvic girdle pain, their symptoms of Pelvic girdle pain decreased compared with the standard group.

One of the advantages of giving craniosacral therapy is that the patient could be monitored whereas in patient who received standard treatment the exercise prescription was given, and results were collected in the follow up visit after two weeks. There was lack of regular monitoring. There were no adverse effects in women who craniosacral therapy which has shown that it is safe to be provided during pregnancy.

The effects of craniosacral therapy have been found to be similar to the effect of manual therapy, myofascial release, massage and acupuncture. Women who received craniosacral therapy expressed that they preferred it over drugs to relieve pain since it was natural and safe. No women dropped out during study and every participant was followed up after the period of two weeks. Studies suggest that mean reduction of $30 \%$ in outcome measure is significant and our results shows reduction in the values of NPRS and PGQ is about 30\% and more indicating it has resulted in positive significant changes (15).

\section{CONCLUSION}

Our study shows that there is reduced pain intensity and improved function, in patients who received craniosacral therapy. The clinical benefits of craniosacral therapy were found to be higher compared to standard treatment for pelvic girdle pain during pregnancy.

\section{CONFLICT OF INTEREST}

Authors declare that there is no conflict of interest.

\section{REFERENCES}

1. Elden, H., Lundgren, I., Robertson, E. Effects of craniosacral therapy as experienced by pregnant women with severe pelvic girdle pain: an interview study. Clinical Nursing studies. 2014; 2(3): 140-151.

2. Nor, L., Östgaard, S., Nielsen, T. F., Östgaard, H. C. Reduction of sick leave for lumbar back and posterior pelvic pain in pregnancy. Spine. 1997 Sep 15; 22(18): 2157-2160.

3. Albert, H. B., Godskesen, M., Korsholm, L., Westergaard, J.G. Risk factors in developing pregnancy-related pelvic girdle pain. Acta obstetricia et gynecologica Scandinavica. 2006 May; 85(5): 539-544.

4. Vleeming, A., Albert, H. B., Östgaard, H. C., Sturesson, B., Stuge, B. European guidelines for the diagnosis and treatment of pelvic girdle pain. European Spine Journal. 2008 Jun; 17(6): 794-819.
5. Firdous, S., Mehta, Z., Fernandez, C., Behm, B., Davis, M. A comparison of Numeric Pain Rating Scale (NPRS) and the Visual Analog Scale (VAS) in patients with chronic cancer-associated pain. Journal of Clinical Oncology. 2017; 35(31): 217

6. Stuge, B., Garratt, A., Jenssen, K. H., Grotle, M. The pelvic girdle questionnaire: a condition-specific instrument for assessing activity limitations and symptoms in people with pelvic girdle pain. Physical therapy. 2011 Jul 1; 91(7): 1096-108.

7. Verstraete, E. H., Vanderstraeten, G., Parewijck, W. Pelvic Girdle Pain during or after Pregnancy: a review of recent evidence and a clinical care path proposal. Facts, views \& vision in Ob Gyn. 2013; 5(1): 33-43.

8. Elden, H., Ladfors, L., Olsen, M.F., Ostgaard, H. C., Hagberg, H. Effects of acupuncture and stabilizing exercises as adjunct to standard treatment in pregnant women with pelvic girdle pain: randomized single blind controlled trial. BMJ. 2005 Mar 31; 330(7494): 761.

9. Elden, H., Östgaard, H.C., Glantz, A., Marciniak, P., Linnér, A.C., Olsén, M.F. Effects of craniosacral therapy as adjunct to standard treatment for pelvic girdle pain in pregnant women: a multicenter, single blind, randomized controlled trial. Acta Obstetricia et Gynecologica Scandinavica. 2013 Jul; 92(7): 775-782.

10. Melzack, R., Wall, P.D. Pain mechanisms: a new theory. Science. 1965 Nov 19; 150(3699): 971-979.

11. Harrison, R. E., Page, J. S. Multi-practitioner upledger craniosacral therapy: descriptive outcome study 2007-2008. The journal of Alternative and Complementary Medicine. 2011 Jan; 17(1): 13-17.

12. Licciardone, J. C., Buchanan, S., Hensel, K. L., King, H. H., Fulda, K. G., Stoll, S. T. Osteopathic manipulative treatment of back pain and related symptoms during pregnancy: a randomized controlled trial. American journal of obstetrics and gynecology. $2010 \mathrm{Jan}$; 202(1): 43.e1-43.e8.

13. Castro-Sánchez, A. M., Mataran-Penarrocha, G. A., Sánchez-Labraca, N., Quesada-Rubio, J. M., GraneroMolina, J., Moreno-Lorenzo, C. A randomized controlled trial investigating the effects of craniosacral therapy on pain and heart rate variability in fibromyalgia patients. Clinical rehabilitation. 2011 Jan; 25(1): 25-35.

14. Petrovic, P., Kalso, E., Petersson, K. M., Andersson, J., Fransson, P., Ingvar, M. A prefrontal non-opioid mechanism in placebo analgesia. Pain. $2010 \mathrm{Jul}$; 150(1): 59-65.

15. Gallagher, E. J., Liebman, M., Bijur, P. E. Prospective validation of clinically important changes in pain severity measured on a visual analog scale. Annals of emergency medicine. 2001 Dec; 38(6): 633-638. 